\title{
Removal of 4-Chlorophenol from Aqueous Media Using Calixpyrrole Modified Silica
}

\author{
Ismail Abbas ${ }^{1}$, Bassem El-Hamaoui ${ }^{1}$, Hilal Njameddine ${ }^{2}$ \\ ${ }^{1}$ Lebanese University \\ Hadath, Beirut, Lebanon \\ ismail.abbas@ul.edu.lb; bassem.elhamaoui@ul.edu.lb \\ ${ }^{2}$ Beirut Arab University \\ Tripoli, Lebanon \\ hilnaj@bau.edu.lb
}

\section{Extended Abstract}

In the present work, we have synthesized new calixpyrrole modified silica followed by characterization with Fourier transform infrared spectroscopy (FTIR), thermal gravimetric analysis (TGA) and scanning electron microscope (SEM) techniques. The behavior of grafted calixpyrrole for the removal of 4-chlorophenol from aqueous media was investigated. Its efficiency was examined through both batch and column extraction methods. The extraction of chlorophenol was carried out as functions of temperature, $\mathrm{pH}$, initial chlorophenol concentration and mass of the adsorbent. Parameters such as initial concentration and quantity of modified silica show a noticeable influence on the uptake capacity of this material. Kinetic studies indicated that the extraction data can be best represented by pseudo second order model. Column extraction data were analyzed through Thomas, Yoon-Nelson and Yan et al models to calculate kinetic coefficients and maximum sorption capacity of the modified silica (III). The extractant can be regenerated by acid treatment without changing its properties.

Keywords: Calixpyrrole Modified Silica, Chlorophenol, Extraction, Kinetics, Thermodynamics, Column Studies. 